\title{
Tafonomía y tecnología lítica en un ambiente altamente dinámico: El caso de la pinguinera del islote La Pastosa (Pcia. de Río Negro) Patagonia, Argentina
}

\author{
Marcelo Cardillo ${ }^{1}$, Eugenia Carranza ${ }^{2}$, Florencia Borella ${ }^{3}$ \\ 1. IMICHIHU Consejo Nacional de Investigaciones Científicas y Técnicas. Saavedra 15. 5to piso. Facultad de \\ Filosofía y Letras. Universidad de Buenos Aires (UBA). Buenos Aires, Argentina. \\ Email: marcelo.cardillo@gmail.com \\ 2. Facultad de Filosofía y Letras. Universidad de Buenos Aires (UBA), Puán 480, Buenos Aires, Argentina. \\ Email: carranza.e89@gmail.com \\ 3. INCUAPA-CONICET. Facultad de Ciencias Sociales, Universidad Nacional de Centro de la Provincia de \\ Buenos Aires (UNICEN), Argentina. Avenida Del Valle 5737 (7400), Olavarría, Buenos Aires, Argentina. \\ Email: fborella@soc.unicen.edu.ar
}

\section{Resumen:}

Este trabajo presenta los primeros resultados de los análisis tafonómicos y tecnológicos realizados sobre el conjunto lítico recuperado en el islote La Pastosa, costa de Río Negro, Patagonia, Argentina (Figura 1). Este registro presenta la particularidad de poseer una alta dinámica formacional ya que se encuentra en un área actualmente ocupada por una colonia de pingüinos Magallánicos (Spheniscus magellanicus). Se realizaron dos muestreos de material lítico sobre los cuales se registraron variables tecnológicas, métricas y tafonómicas.

La Pastosa es el islote de 8 Ha que se ubica a $768 \mathrm{~m}$. del continente, y sólo queda unido a éste durante la bajamar por una estrecha franja de tierra que atraviesa una marisma. Si bien se desconoce la edad absoluta de este islote, sabemos que a partir del Holoceno tardío, alrededor del 3000 A.P. se habría estabilizado el nivel de costa (Favier Dubois 2013), y ya para ese entonces fue visitado por cazadores- recolectores, quienes realizaron allí inhumaciones humanas en la parte más elevada (Favier Dubois et al. 2009). En la actualidad, este espacio presenta una gran diversidad faunística, hallándose en el sector litoral noreste del islote (frente al mar abierto), un apostadero reproductivo de lobos marinos de un pelo (Otaria flavescens) de reciente formación (Svendsen et al. 2009: 8), así como una gran variedad de aves marinas).

Las tareas de muestreo se centraron en el sector de mayor densidad de artefactos líticos, cercana en la actualidad al área central del islote (Figura 1). El contexto de recuperación de los materiales puede ser dividido en tres categorías: A) sectores de tránsito de los pingüinos, que conforman senderos libres de vegetación; B) la boca de las madrigueras y C) espacios marginales que se corresponden a los sectores de acumulación de desechos (pequeñas ramas y pasto seco, plumas, excrementos, carcasas de pingüino) en los márgenes de la zona de tránsito (Figura 2).

Durante la recolección se registró la posición original de los artefactos, o cara de recuperación, así como el contexto del hallazgo. Posteriormente, en el laboratorio se relevaron variables tipológicas (instrumento, núcleo, desecho de talla), materia prima; así como métricas (volumen en $\mathrm{mm}^{3}$ ) y

Published by the School of History, Classics and Archaeology, University of Edinburgh ISSN: 2055-0472. URL: http://journals.ed.ac.uk/lithicstudies/

This work is licensed under a Creative Commons Attribution 2.5 UK: Scotland License. 
tafonómicas. Particularmente estas últimas fueron utilizadas para caracterizar y comparar ambos conjuntos.

El registro y análisis de las variables tafonómicas indicadoras de las alteraciones es una línea de investigación para aproximarse a la intensidad de las transformaciones físicas del contexto, indicando a su vez el grado de integridad del mismo (Schiffer 1983; Burroni et al. 2002). Las variables consignadas fueron: el estadio de alteración producto de la meteorización física de la pieza por cara (W: W0, W1, W2, W3); la presencia por cara de concreción de carbonato en porcentaje; y el estado de integridad de la pieza (entero o fragmentado).

Los resultados obtenidos muestran que a través del tránsito por determinados sectores y la excavación de los nidos, los pingüinos exponen material sepultado (Figura 5.1), el que es desplazado a través de las vías de circulación de estos mismos animales (Figura 5.2), pudiendo ser luego redepositado en sectores marginales de circulación (Figura 5.3). Tal como se mencionó éstos son principalmente pequeños parches de vegetación y desechos orgánicos, que poseen una estabilidad relativa alta. Por este motivo creemos que en estos parches, los materiales tienen mayor posibilidad de acumularse y tal vez reingresar al sustrato. La evidencia preliminar obtenida para esta área de estudio sugiere que el registro lítico está en continua conformación, en este caso a partir de la acción de los pingüinos y si bien el establecimiento de esta colonia es reciente, su impacto en el registro arqueológico es muy significativo.

Palabras clave: Costa Norpatagónica; tafonomía lítica; pingüinos; procesos de formación natural; Islote la Pastosa

\section{Introducción}

El islote La Pastosa es un interesante caso de estudio sobre la conformación del registro lítico, ya que se trata de un espacio acotado actualmente sujeto a intensa faunaturbación, generada por la reciente formación de una colonia de pingüinos patagónicos (Spheniscus magallanicus). Desde el punto de vista tecnológico este sector representa un espacio particular, con evidencias de uso no sólo con fines económicos sino rituales, por parte de grupos cazadores-recolectores que habitaron el área, al menos desde el 3000 A.P., ya que en ésta se han identificado enterramientos humanos en distintos sectores (Favier Dubois et al. 2007; Favier Dubois et al. 2009).

La información disponible sobre los grupos humanos del área, proviene del trabajo sistemático en la costa oeste de Río Negro, a partir de las evidencias obtenidas de la implementación de estudios distribucionales del material superficial (Manzi et al. 2011), así como de excavaciones sistemáticas. Esta evidencia señala que el área estuvo ocupada por grupos cazadores-recolectores y pescadores entre los 6000-400 años A.P., que hicieron uso prolongado de los recursos costeros a lo largo del tiempo, en particular mamíferos marinos como el lobo marino; peces, moluscos y recursos terrestres como el guanaco (Borella et al. 2011).

Esta costa se caracteriza además por la relativa heterogeneidad en su geomorfología y en la distribución de recursos, lo que en parte se refleja en la desigual distribución de los elementos arqueológicos (Favier Dubois \& Borella 2011). El área de estudio presenta la particularidad de ser una costa baja con arrecifes en barrera y poseer ambientes de humedales combinados con afloramientos rocosos, alguno de los cuales constituyen islotes que albergan gran diversidad de colonias reproductivas de aves y mamíferos marinos.

La tecnología de los grupos humanos de este sector está representada principalmente a través del registro lítico, aunque recientemente hemos recuperado algunos tiestos cerámicos, lo que está señalando una ocupación más tardía respecto a la inicialmente conocida para el área, a partir del fechado de los restos humanos recuperados en superficie en La Pastosa (Favier Dubois et al. 2007). El material lítico se caracteriza por ser básicamente de tipo 
expeditivo, donde predominan instrumentos de filo simple como denticulados, de retoque sumario (sensu Aschero 1975; 1983) y raspadores. En algunos sectores cercanos al área de estudio, se ha recuperado también bolas líticas y pesas de línea o red (Favier Dubois \& Scartascini 2012), potencialmente vinculadas a la pesca. En proporciones menores se han recuperado puntas de proyectil y bifaces de variada morfología, que sugieren cambios a lo largo del tiempo en las estrategias y/o tecnologías de caza (Cardillo \& Scartascini 2007; Cardillo \& Favier Dubois 2011).

En el área de estudio, las excavaciones recientes sugieren estrategias tecnológicas similares, con un énfasis en el empleo de materias primas de calidad buena y muy buena para la talla, no disponibles de forma inmediata y transportadas de áreas aledañas (Alberti 2015: 256). Sin embargo su densidad, composición y distribución es variable en el espacio, lo que sugiere una jerarquización de determinados espacios por parte de los grupos humanos. Asimismo, la particularidad del islote -como un espacio acotado de accesibilidad limitadasugiere diferencias en relación a los demás sectores ubicados a lo largo de la franja costera, lo que podría reflejarse en el modo de uso a lo largo del tiempo y por ende, en las estrategias tecnológicas allí implementadas.

Por otro lado, la alta dinámica de este espacio, ocupado actualmente por una creciente colonia de pingüinos Magallánicos, plantea la importancia de tener en cuenta los aspectos tafonómicos y formacionales del registro arqueológico.

Por este motivo, así como por la ubicuidad y distribución del registro lítico en el área de estudio, se ha comenzado a aplicar una perspectiva tafonómica, la cual provee información sumamente útil del proceso de formación de los conjuntos, así como de la dinámica de los paisajes estudiados, como se verá a continuación.

\section{2. Área de estudio}

La Pastosa es el islote de mayor superficie de los que conforman el complejo Islote Lobos, con aproximadamente $8 \mathrm{Ha}$. Está formada básicamente por afloramientos graníticos que han resistido la acción erosiva del mar y bloques sueltos de calizas duras, sobre los que se han formado médanos semifijos (Sánchez 1973: 112-141). Presenta una vegetación arbustiva achaparrada y abierta que incluye: Atriplex spp., Lycium chilense, Larrea spp., Cyclolepis genistoides, Suaeda divaricata y Stipa tenuis (Gonzalez et al. 1998). El área intermareal -que conecta el Islote con el continente- está constituida por el contacto entre ambos afloramientos (graníticos y de calizas duras de edad Rocanense, que lo cubren parcialmente), formando numerosos pozos de marea de profundidad variable (piletones o marmitas) (Sánchez 1973: 112-141). Mientras que en el perímetro litoral del islote se observa la acreción de sedimentos arenosos, guijarros de variado tamaño y acumulación de banco de conchillas (mitílidos) y huesos de pinnípedos (Borella \& Borrero 2010), por lo que es esperable que el islote haya podido ser un poco más pequeño en el pasado. La Pastosa se ubica a $768 \mathrm{~m}$. del continente, y sólo queda unido a éste durante la bajamar por una estrecha franja donde se desarrolla la marisma, con una vegetación compuesta por: Spartina densiflora y Salicronia ambigua (González et al. 1998). Si bien se desconoce la edad absoluta de este islote, sabemos que a partir del Holoceno tardío, alrededor del 3000 A.P. se habría estabilizado el nivel de costa (Favier Dubois 2013), y ya para ese entonces fue visitado por cazadores- recolectores, quienes realizaron allí inhumaciones humanas en la parte más elevada (Favier Dubois et al. 2007; Favier Dubois et al. 2009). En la actualidad, este espacio presenta una gran diversidad faunística, hallándose en el sector litoral noreste del islote (frente al mar abierto), un apostadero reproductivo de lobos marinos de un pelo (Otaria flavescens) de reciente formación (Svendsen et al. 2009: 8), así como una gran variedad de aves marinas (pingüinos, gaviotas y gaviotines). 
La Pastosa es el más elevado de los islotes, presenta una forma de cono truncado, lo que permite conjeturar que el sector central y el área periférica aledaña, son las zonas más antiguas del islote y es donde se concentra el registro arqueológico antes mencionado material lítico y restos bioarqueológicos - (Figura 1).
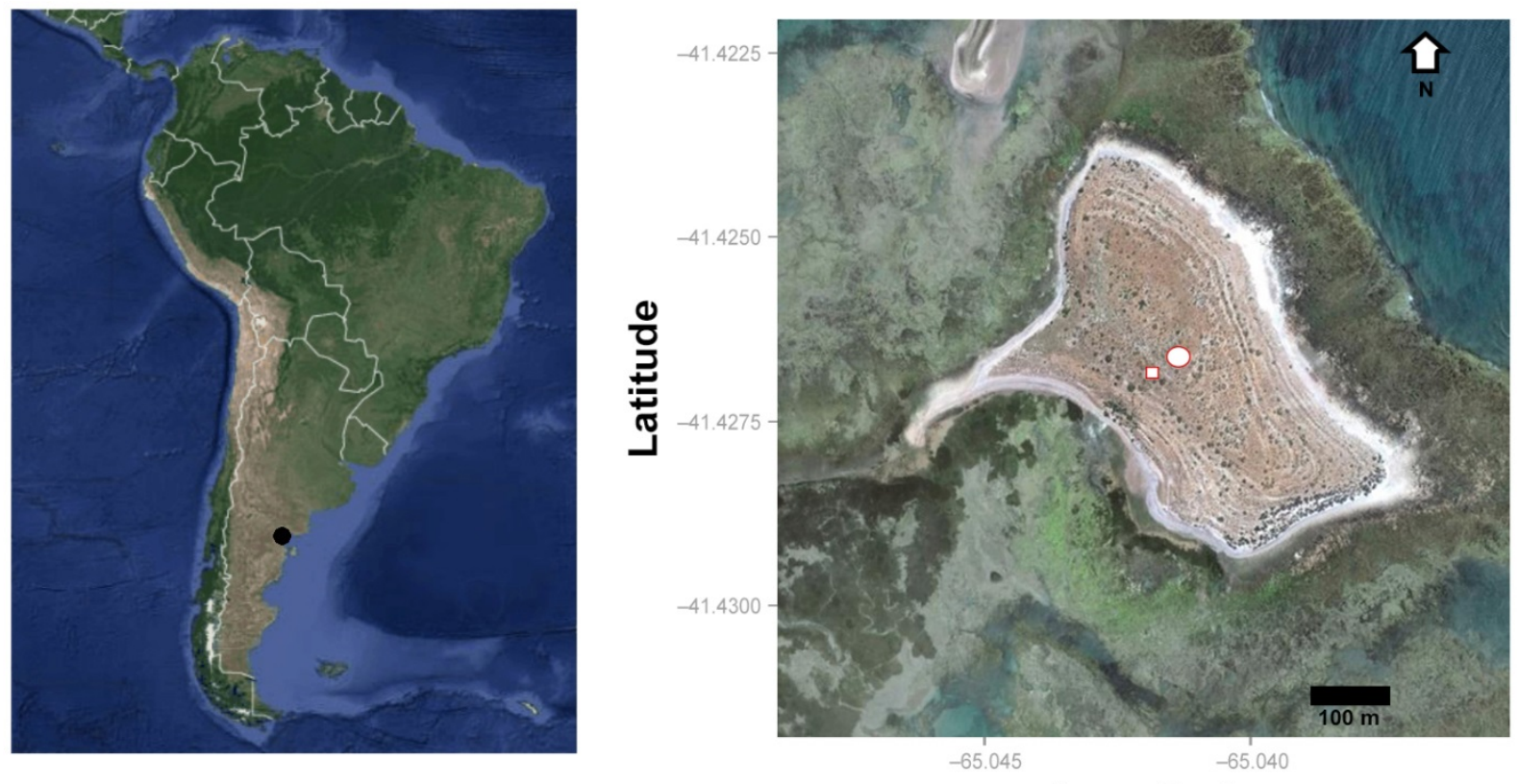

\section{Longitud}

Figura 1. Izquierda, Mapa de Sudamérica con la localización del área de estudio. Derecha, Isla la Pastosa. Círculo: sector del muestreo 1 (S1). Cuadrado, sector del muestreo 2 (S2). El tamaño es relativo al área de recolección. (Para imágenes de mayor resolución, consulte los archivos suplementarios.)

Figure 1. On the left, Map of South America. The location of the study area is shown. On the right, La Pastosa Islet. Circle: sampling sector 1 (S1). Square: sampling sector 2 (S2). Size is relative to the collection area. (For higher resolution images, see the supplemental files.)

\section{Los pingüinos como agentes tafonómicos}

Tal como se mencionó en la introducción, el islote constituye un área de reproducción reciente del pingüino de Magallanes (Spheniscus magellanicus), ave marina no voladora de unos 3,5 a $4 \mathrm{~kg}$ de peso promedio con una altura que no supera los $70 \mathrm{~cm}$. Estimaciones actuales sugieren que su número se está incrementando, y se han contabilizado más de 1000 parejas (Bertellotti 2013: 1-59), mientras que en el censo realizado en el año 2002 por investigadores del Centro Nacional Patagónico se habían detectado sólo 22 nidos (Schiavini et al. 2005).

Observaciones realizadas en diversos trabajos de campo (2006, 2007 y 2015) permiten plantear que, efectivamente, el área de nidificación se ha extendido en los últimos años, ocupando en la actualidad casi la totalidad de la superficie del islote. Estas aves monógamas conforman la misma pareja año tras año, utilizando el nido ocupado en la temporada anterior, o uno muy cercano (Bertellotti 2013: 1-59). Así, el uso reiterado de sus nidos a lo largo de su ciclo de vida provoca un gran impacto sobre la superficie del terreno, especialmente en un área tan limitada como ésta.

Estas aves suelen armar sus nidos debajo de arbustos, en cuevas o madrigueras excavadas en el suelo, o también a cielo abierto, sin cobertura alguna (Schiavini et al. 2005). En el caso del Islote La Pastosa los nidos han sido excavados y presentan grandes dimensiones, conformando madrigueras que se comunican entre sí. El registro de las dimensiones de los túneles excavados por esta especie, alcanzan un poco menos del metro de longitud y hasta 1-2 
metros de profundidad, mientras que la remoción de sedimentos de substrato sería de unos $0,05 \mathrm{~m}^{3}$ (Stokes \& Boersma 1991).

La relevancia de esta especie como agente tafonómico en la costa patagónica fue evaluada por I. Cruz, particularmente en relación al registro óseo (Cruz 1999; 2004; 2007). Esta autora observó que los pingüinos constituyen importantes agentes erosivos en la costa del continente a partir del tránsito constante desde sus nidos hacia el mar. La circulación de estas aves genera senderos, provocando el desplazamiento del material arqueológico depositado en superficie, así como la acumulación en determinados puntos. Por otra parte, al cavar los nidos alteran la cobertura vegetal, remueven artefactos y contribuye a la conformación de palimpsestos (Cruz 2004, 2007).

La excavación de los nidos y la consecuente remoción de sedimentos provocan cierta inestabilidad y el colapso de las superficies aledañas. Esta situación de transporte, redepositación y erosión de espacios asociados a áreas de nidificación de aves marinas ha sido registrada en otros contextos donde se ha descrito pisoteo, acumulación de plumas y abonos, junto con la destrucción de la vegetación (Butler 1995: 44-50).

\section{Métodos y análisis}

Las tareas de muestreo se centraron en el sector de mayor densidad de artefactos líticos, próxima actualmente al área central del islote (Figura 1). El contexto de recuperación de los materiales puede ser dividido en tres categorías: A) sectores de tránsito de los pingüinos, que conforman senderos libres de vegetación; B) la boca de las madrigueras y C) espacios marginales que se corresponden a los sectores de acumulación de desechos (pequeñas ramas y pasto seco, plumas, excrementos, carcasas de pingüino) en los márgenes de la zona de tránsito (Figura 2).

Se realizaron dos muestreos en sectores cercanos al centro del islote en donde se detectaron materiales en superficie. El perímetro de estos muestreos está separado por unos $32 \mathrm{~m}$, pero se encuentran en contextos topográficos diferentes.

El primero de ellos, denominado Sector 1 (S1) (Figuras 1 \& 3.1), comprende un área de $2.615 \mathrm{~m}^{2}$ con una densidad de 0.01 artefactos por $\mathrm{m}^{2}$ y se localiza hacia el centro del islote, en un espacio con relieve escaso y cobertura vegetal media, básicamente compuesta por el arbusto jarilla (Larrea divaricata). Este sector se caracteriza por la alta frecuencia de nidos y madrigueras. El segundo sector (S2) (Figuras 1 \& 3.2), de menor tamaño, comprende un área de $154 \mathrm{~m}^{2}$ con una densidad de 0.18 artefactos por $\mathrm{m}^{2}$ y se ubica a lo largo de una pendiente de unos $45^{\circ}$ al borde de una duna, con una cobertura vegetal similar y una frecuencia de nidos relativamente menor, aunque esta variable no pudo ser cuantificada en detalle.

Durante la recolección se registró la posición original de los artefactos, o cara de recuperación, así como el contexto del hallazgo. Posteriormente, en el laboratorio se relevaron variables tipológicas (instrumento, núcleo, desecho de talla), materia prima; así como métricas (volumen en $\mathrm{mm}^{3}$ ) y tafonómicas. Particularmente estas últimas fueron utilizadas para caracterizar y comparar ambos conjuntos.

El registro y estudio de las variables tafonómicas indicadoras de las alteraciones es una línea de investigación para aproximarse a la intensidad de las transformaciones físicas del contexto, indicando a su vez el grado de integridad del mismo (Schiffer 1983; Burroni et al. 2002). Las variables consignadas fueron: el estadio de alteración producto de la meteorización física de la pieza por cara (W: W0, W1, W2, W3); la presencia por cara de concreción de carbonato en porcentaje; y el estado de integridad de la pieza (entero o fragmentado).

Éstas nos dan claves para entender la estabilidad de los artefactos en el medio y pueden aplicarse para ponderar los efectos de los procesos tafonómicos sobre los conjuntos. Así, el cambio de posición y estabilidad de los artefactos permitirá evaluar la estabilidad relativa, 
desde su descarte hasta su posterior recuperación. Por estabilidad se entiende el estudio de varios procesos tafonómicos que actuaron sobre el registro (Borrero 2004; Borrazzo 2006), variable que es evaluable mediante el análisis de las modificaciones espaciales de los conjuntos. Ésta es una forma de aproximarse al estudio de las alteraciones postdepositacionales producto de la interacción de diversas variables: pendiente, vegetación, viento, tipo de substrato, entre otras.

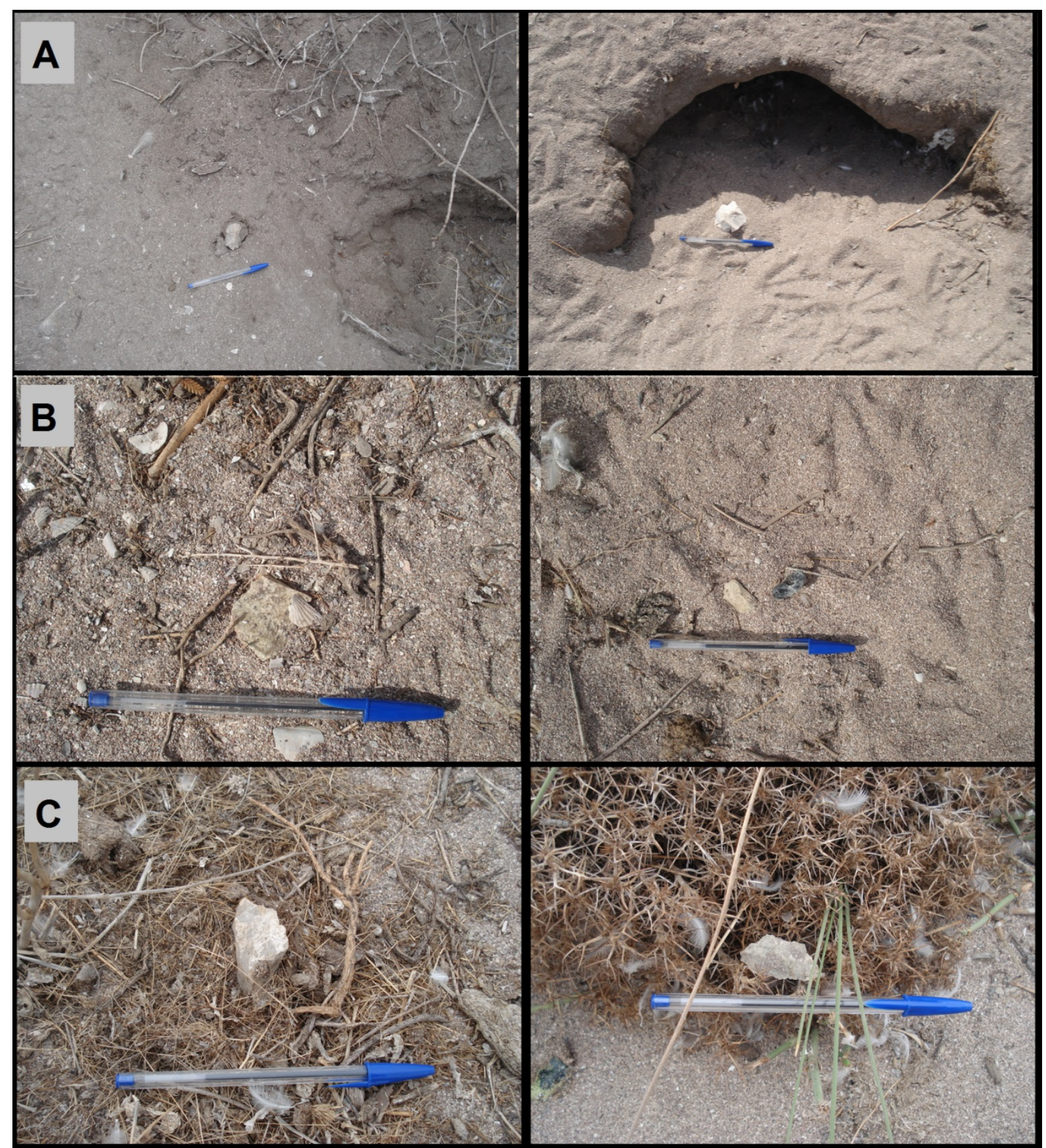

Figura 2. Tres tipos de entornos donde se recuperaron materiales, vinculados a la acción de los pingüinos. A: madrigueras. B: sectores de tránsito o senderos. C: sectores marginales.

Figure 2. Three types of environments where materials were recovered linked to the action of penguins. A: burrows. B: transit areas or trails. C: marginal sectors. 


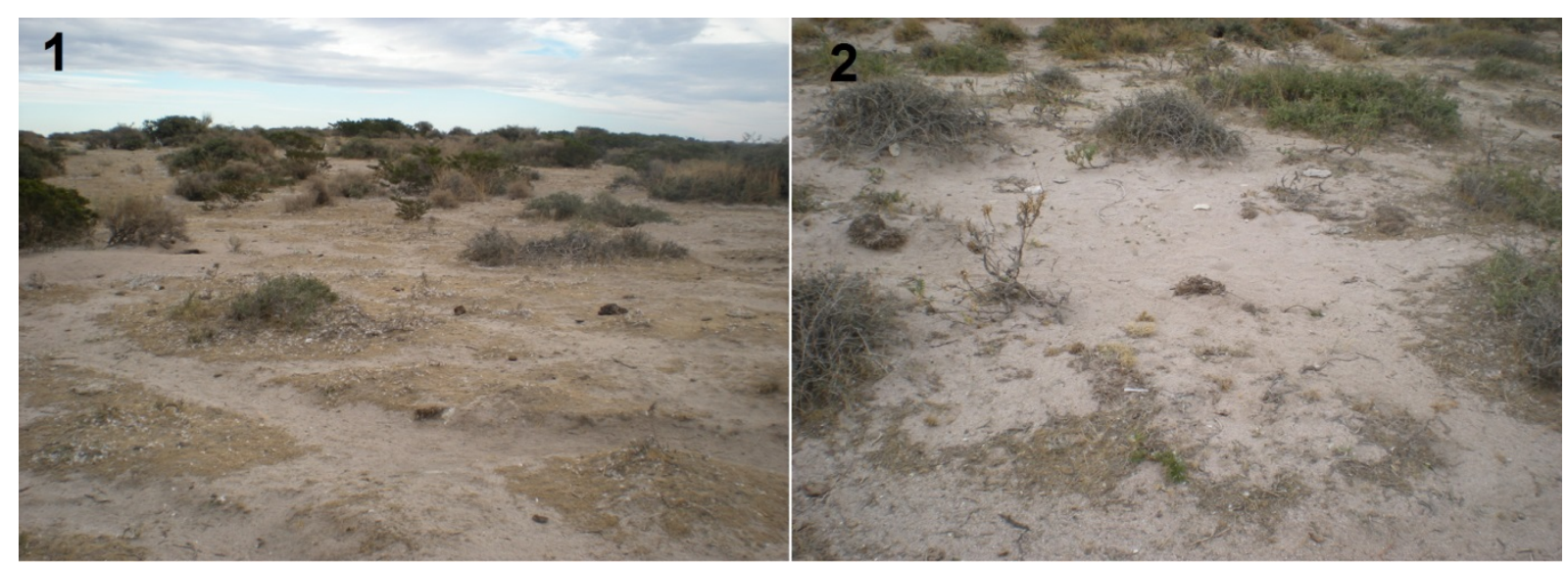

Figura 3. Sectores muestreados. 1) Sector 1, se observan claramente los senderos formados por el tránsito de los pingüinos y las áreas de acumulación de desechos vegetales y animales, conformando parches. 2) Sector 2, visto desde la base de la pendiente. (Para imágenes de mayor resolución, consulte los archivos suplementarios.)

Figure 3. Sectors sampled. 1) Sector 1, clearly showing the paths formed by the transit of penguins and the accumulation areas of plant and animal waste, forming patches. 2) Sector 2, a view from the base of the slope. (For higher resolution images, see the supplemental files.)

El material fue recolectado siguiendo el protocolo de análisis tafonómico (Borrazzo 2006; Carranza 2015: 20-25) donde se consigna la cara de recuperación (Figura 4). Los artefactos fueron levantados en el campo registrando la superficie expuesta en el momento de su recuperación para reconstruir su historia deposicional. Se distinguió en las lascas entre: (A) para la cara de recuperación; (B) para la cara en contacto con la superficie del suelo. En los ecofactos, los núcleos y los desechos no diferenciados, se distinguió entre: (C) cara de recuperación; (D) para la superficie en contacto con el suelo. Siguiendo el protocolo de análisis propuesto por Borrazzo (2006) se descartaron aquellas piezas que presentaban en una de sus caras un porcentaje del $100 \%$ de corteza, fueron eliminadas del análisis debido a la meteorización física diferencial que registra la superficie de la corteza, la cual produciría un sesgo que no permitiría distinguir la historia deposicional de la pieza de sus huellas de meteorización (Borrazzo 2006).

La alteración producto de la meteorización física es producida mayormente por el impacto de partículas sedimentarias transportadas por agentes como el agua o el viento. Es un tipo de meteorización que afecta a la morfología de los artefactos produciendo: redondeamiento de aristas, pulidos y/o brillos superficiales (Camuffo 1995). Los efectos de la meteorización física están condicionados por factores exógenos: la disponibilidad de agua/viento, la temperatura, la pendiente y la disponibilidad de sedimentos sueltos, por ejemplo y endógenos como: la mineralogía de las rocas, el tamaño de grano, las grietas o fracturas internas, la dureza y la isotropía (Dorn 2009).

Otro parámetro considerado ha sido la presencia de rock coating, principalmente la carbonatación, que consiste en un proceso acrecional y deposicional de cristales minerales de carbonatos de calcio (Borrazzo 2009; Dorn 2009). Estimar su presencia en los artefactos recuperados en los muestreos es un indicador útil de la estabilidad relativa de la pieza, ya que muestra que la misma permaneció sin moverse el tiempo suficiente en condiciones adecuadas para que se forme dicha concreción.

Con el objetivo de comparar entre sectores se realizó una cuantificación de la muestra y se compararon las frecuencias observadas en cada caso mediante el test de $\mathrm{Chi}^{2}$, la estimación de la probabilidad asociada se realizó mediante la aproximación asintótica de Monte Carlo (Brown \& Rothery 1993: 539-540), más apropiada para aquellos casos en los que se observan frecuencias muy bajas (menores a cinco, o nulas). El volumen fue comparado mediante el test $t$ de Student, tras verificar el cumplimiento del supuesto de heterocedasticidad de la varianza. 
La cuantificación general y la comparación entre conjuntos se realizó mediante el programa Past (Hammer et al. 2001).
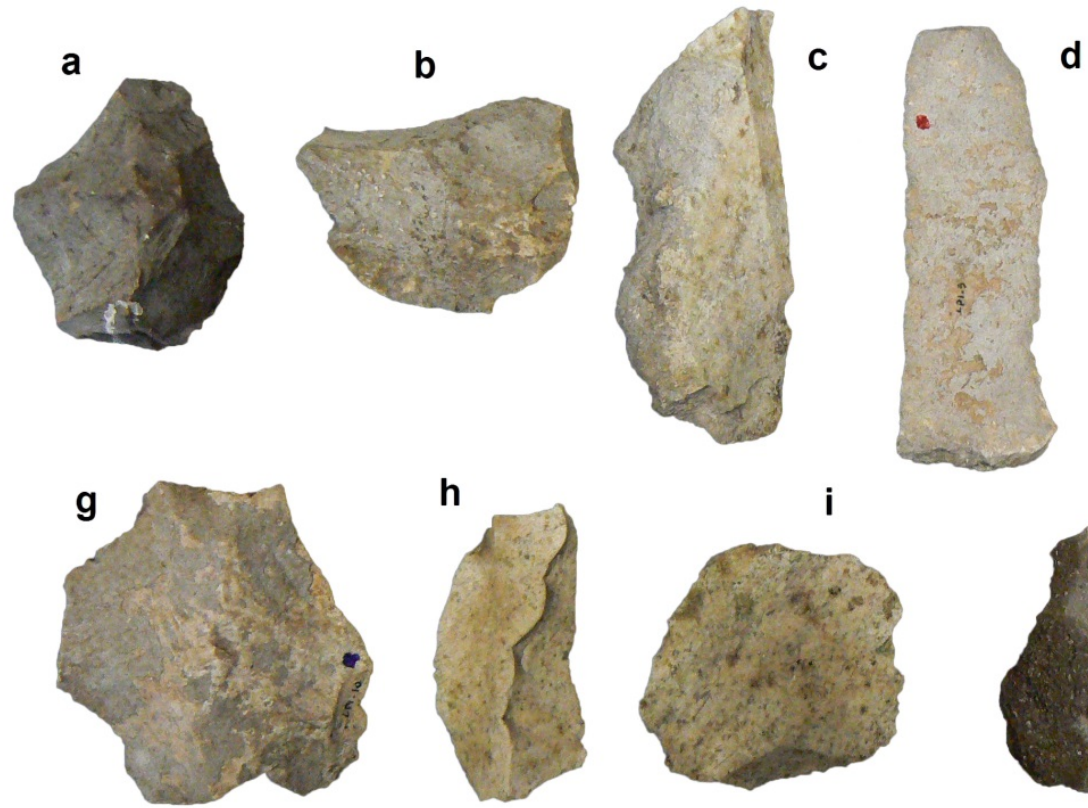

d e
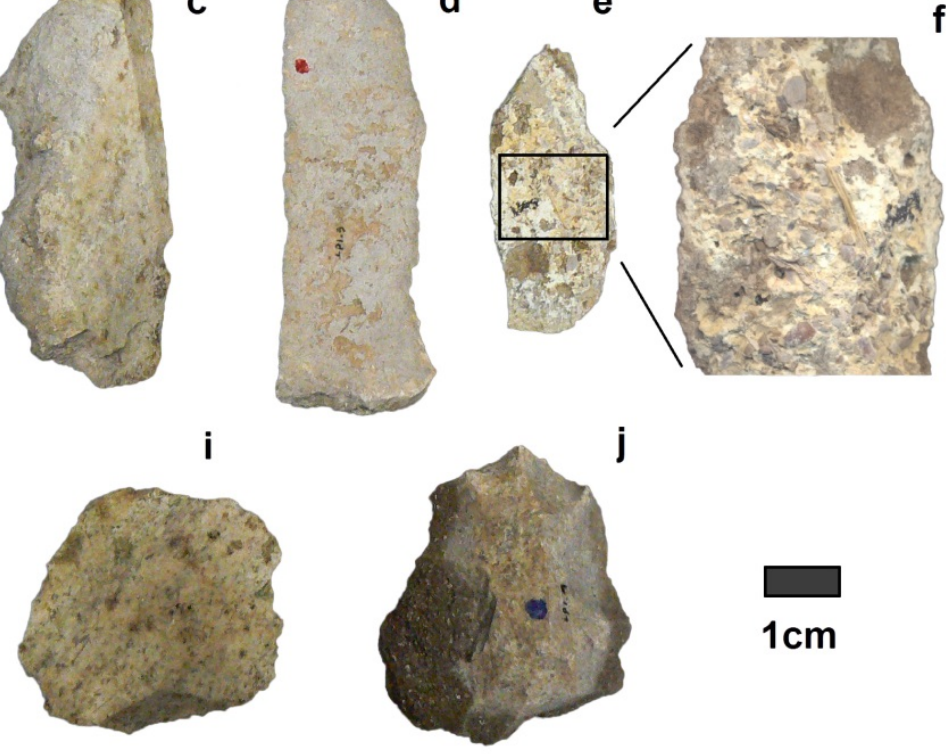

j

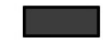

$1 \mathrm{~cm}$

Figura 4. Artefactos recuperados en los muestreos. Sector 1: a. Núcleo poliédrico con carbonatación total sobre su superficie; d. Hoja con carbonatación total en ambas caras; g. Lasca angular con meteorización y carbonatos sobre su superficie; h. Lasca angular con elevada meteorización; j. Núcleo discoidal irregular con carbonatación total sobre su superficie. Sector 2: b. Lasca angular con carbonatación sobre su superficie; c. Lasca de dorso con carbonatación parcial en ambas caras; e. Lasca angular; f. detalle de la superficie con adherencias de carbonatos y restos vegetales; i. Lasca angular con meteorización física parcial y adherencia de moho sobre su superficie. (Para imágenes de mayor resolución, consulte los archivos suplementarios.)

Figure 4. Sampled stone artifacts. Sector 1: a. Polyhedral core with carbonation covering all of its surface; d. Laminar flake with total carbonation on both sides; g. Angular flake with weathering and carbonates on its surface; h. Angular flake with high weathering; j. Amorphous core with complete carbonation on its surface. Sector 2: b. Angular flake with carbonation on its surface; c. Backed flake with partial carbonation on both sides; e. Angular flake; f. surface detail with adhesions of carbonates and vegetal debris; i. Angular flake with partial physical weathering and rust on its surface. (For higher resolution images, see the supplemental files.)

\section{Resultados}

\subsection{Meteorización física}

Los estadios de meteorización física observados para ambas caras (A y B) son semejantes entre ambos sectores. El S1 posee $n=19$ (A, B) lo que corresponde a un $63 \%$ del total de esta muestra; mientras que el S2 en el estadio más bajo (W0) entre $n=18$ (A) y n=20 (B) (67 a $74 \%$ ). En el estadio más alto (W3), la meteorización física para el S1 es de entre $n=3$ (A) y n=4 (B) (10 a 13\%); mientras que es nula para el S2 (Tabla 1, Figura 4).

Tabla 1. Frecuencias absolutas y relativas de meteorización física por sector.

Table 1. Absolute and relative frequencies of physical weathering per sector.

\begin{tabular}{lccccccccc}
\hline A & W0 & \% & W1 & \% & W2 & \% & W3 & \% & Total \\
\hline Sector 1 & 19 & 63.33 & 6 & 20 & 2 & 6.67 & 3 & 10 & 30 \\
Sector 2 & 18 & 66.67 & 9 & 33.33 & & 0 & 0 & 0 & 27 \\
B & W0 & W0 (\%) & W1 & W1 (\%) & W2 & W2 (\%) & W3 & W3 (\%) & \\
Sector 1 & 19 & 63.33 & 5 & 16.67 & 2 & 6.67 & 4 & 13.33 & 30 \\
Sector 2 & 20 & 74.07 & 7 & 25.93 & 0 & 0 & 0 & 0 & 27 \\
\hline
\end{tabular}


Esto sugiere que se registra una meteorización física levemente mayor en el sector 1, aunque las diferencias son en general, muy pequeñas y no significativas (Cara $\mathrm{A}: \mathrm{Chi}^{2}=5.48$, $\mathrm{p}>0.05$, Cara B: $\mathrm{Chi}^{2}=6.21, \mathrm{p}>0.05$ ).

\subsection{Carbonatación}

La ocurrencia de carbonatación es distinta en ambos muestreos, en el S1, sólo un 23\% no presenta acreción, mientras que cerca del $47 \%$ la presenta en ambas caras. En el S2, en cambio, la carbonatación presenta una frecuencia menor. En el S2 un 26\% presenta esta modificación postdepositacional en ambas caras (Tabla 2), mientras que el 63\% no está carbonatada.

Tabla 2. Frecuencias absolutas y relativas de carbonatación por sector.

Table 2. Absolute and relative carbonation frequencies per sector.

\begin{tabular}{lccccccc}
\hline Carbonatación & Ambas & $\%$ & Una & $\%$ & Ninguna & $\%$ & Total \\
\hline Sector 1 & 14 & 46.67 & 9 & 30 & 7 & 23.33 & 30 \\
Sector 2 & 7 & 25.93 & 3 & 11.11 & 17 & 62.96 & 27 \\
\hline
\end{tabular}

En ambos casos la carbonatación en sólo una cara es la situación menos frecuente y oscila entre un $30 \%$ y un $11 \%$ respectivamente.

El test de $\mathrm{Chi}^{2}$ sobre la tabla de contingencia indica que estas diferencias son significativas $\left(\mathrm{Chi}^{2}=9.36, \mathrm{p}=0.009\right.$ ), lo que sostiene la afirmación de que ambos sectores constituyen contextos con condiciones de alteración diferentes (Figura 4).

\subsection{Estado de las piezas}

El análisis de piezas enteras y fragmentadas por sector arrojó resultados no significativos. Para el S1 se cuantificó una frecuencia de 50\% de piezas enteras y de piezas fragmentadas. Para el S2, los resultados indican un 52\% de piezas enteras y un $48 \%$ de fragmentadas. Estos resultados sugieren que existen probabilidades equivalentes de fractura.

\subsection{Clases artefactuales}

En cuanto a las categorías artefactuales recuperadas, en ambos sectores predominan los desechos (Figura 4). En el S1 la frecuencia de desechos es de $n=21$ (70\%), se recuperaron además instrumentos y núcleos (Tabla 3). En el S2, en cambio, los desechos son la única categoría presente. Esto implica claramente diferencias entre ambos sectores $\left(\mathrm{Chi}^{2}=9.61\right.$, $\mathrm{p}=0.02$ ); sin embargo, dada la cercanía espacial y el tamaño pequeño de las muestras, estos resultados se deben tomar con precaución. Es más, las características de los desechos del S2, así como la materia prima (chert), sugieren que se trata de material procedente de un mismo núcleo, por lo que parecen proceder de la misma secuencia de reducción, lo que cobra especial interés si se toma en cuenta la dispersión de estos materiales.

Tabla 3. Frecuencias absolutas y relativas de categoría de artefacto por sector. Table3. Absolute and relative frequencies of artifact category per sector.

\begin{tabular}{lccccccccc}
\hline Sector & Desechos & $\%$ & Ecofacto & $\%$ & Instrumento & $\%$ & Núcleo & $\%$ & Total \\
\hline S1 & 21 & 70 & 1 & 3.33 & 4 & 13.33 & 4 & 13.33 & 30 \\
S2 & 27 & 100 & 0 & 0 & 0 & 0 & 0 & 0 & 27 \\
\hline
\end{tabular}




\subsection{Volumen}

Por último, la comparación entre ambos conjuntos señala que no existen diferencias de volumen entre los conjuntos ( $\mathrm{t}=0.31, \mathrm{p}>0.05$ ), el muestreo de $\mathrm{S} 1$ posee un intervalo que oscila entre 1.32 y $65.28 \mathrm{~cm}^{3}$ con una media de $19 \mathrm{~cm}^{3}$. En el sector de la duna, S2, los valores son ligeramente más amplios de 1 a $102.6 \mathrm{~cm}^{3}$ y un promedio de $16.65 \mathrm{~cm}^{3}$. Esto sugiere que la probabilidad de exposición y desplazamiento en relación a esta variable, es semejante en ambos sectores.

\section{Discusión y conclusiones}

La evidencia preliminar obtenida para esta área de estudio sugiere que el registro lítico está en continua conformación, en este caso a partir de la acción de los pingüinos y si bien el establecimiento de esta colonia es reciente, su impacto en el registro arqueológico es muy significativo.

La alta proporción de materiales con depósitos de carbonatos, así como de adherencias orgánicas en el S1 sugiere que el material allí recuperado posee una exposición más reciente respecto al del S2. Además, sugiere que el sustrato que lo contenía previa a la exposición presenta condiciones físico-químicas que permiten la formación de precipitados sobre la superficie de las piezas mientras éstas estuvieron contenidas en la matriz sedimentaria.

A través del tránsito por determinados sectores y la excavación de los nidos, los pingüinos exponen material sepultado (Figura 5.1), el que es desplazado a través de las vías de circulación de estos mismos animales (Figura 5.2), pudiendo ser luego redepositado en sectores marginales de circulación (Figura 5.3), tal como se mencionó éstos son principalmente pequeños parches de vegetación y desechos orgánicos, que poseen una estabilidad relativa alta. Por este motivo creemos que en estos parches, los materiales tienen mayor posibilidad de acumularse y tal vez reingresar al sustrato.

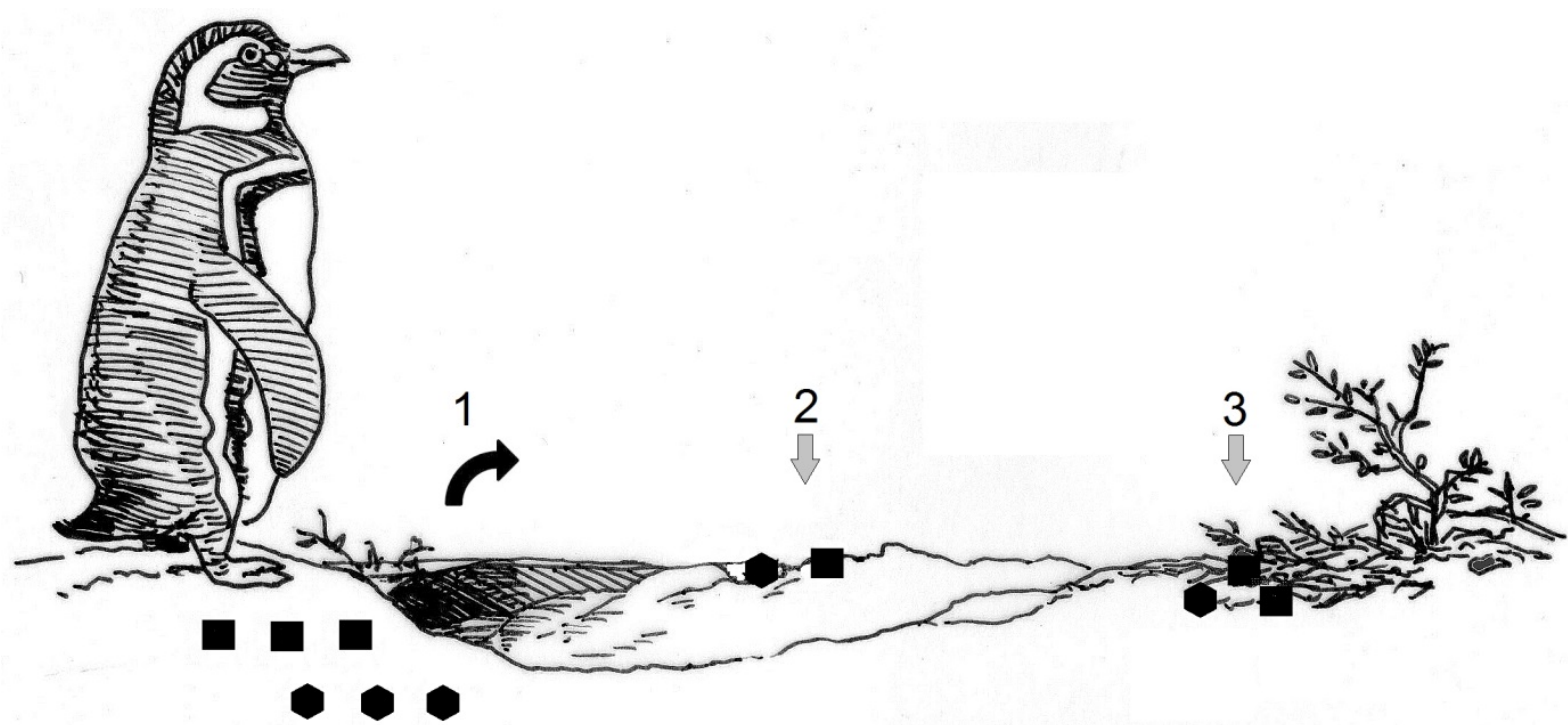

Figura 5. Modelo tafonómico para el conjunto estudiado. En el sector del interior (S1) los pingüinos son el principal agente de remoción de los conjuntos (cuadrados, hexágonos, 1), sectores de tránsito (2), áreas marginales que tienden a concentrar hallazgos, conformando palimpsestos (3).

Figure 5. Taphonomic model for the studied assemblage. In the interior sector (S1) penguins are the main agent of artifact removal (squares, hexagons, 1), transit sectors (2), marginal areas that concentrate archaeological material, forming palimpsests (3).

En el caso del segundo muestreo (S2), la mayor frecuencia de material sin carbonatación sugiere un mayor tiempo de exposición, lo que habría hecho que las partículas de arena 
arrastradas por el viento "limpien” la superficie de las piezas. Esta exposición también podría vincularse a la amplia distribución de los desechos, posiblemente pertenecientes a una misma secuencia de reducción.

De acuerdo a datos obtenidos en la pingüinera de Punta Tombo (Chubut) los nidos excavados pueden tener una profundidad promedio variable entre $62,9 \mathrm{~cm}$ a $23 \mathrm{~cm}$ (Schiavini et al. 2005). En relación a ésto observamos -parcialmente expuesta en un perfil de médanouna delgada lente de valvas y carbones (conchero) de no más de $10 \mathrm{~cm}$ de espesor, sepultada a unos $5 \mathrm{~cm}$ del suelo actual, visible a raíz de la erosión generada por la acción de los pingüinos. Considerando las dimensiones que pueden alcanzar las madrigueras (profundidad y extensión) se evidencia claramente la magnitud y el grado de perturbación del substrato que puede generar la acción de estas aves marinas en las áreas escogidas para la nidificación.

En cuanto a las demás variables estudiadas, éstas sugieren regularidades en la composición del registro en ambas áreas, lo que señala la importancia del estudio del proceso de formación y distribución espacial de los conjuntos. Si bien el esquema presentado es básico, otras trayectorias son posibles, ya que por ejemplo, los sectores de tránsito generan franjas de erosión que pueden activar procesos de deflación y por ende, exponer materiales.

Asimismo, como se observó en el S2, localizado al borde de una duna, estos espacios parecen ser muy sensibles a sucesivos eventos de enterramiento y exposición por erosión. Este sencillo modelo puede ser puesto a prueba mediante estudios experimentales. De esta manera se podrían platear pistas experimentales en contextos cercanos a madrigueras, o sectores de tránsito, (véase por ejemplo: Borrazzo 2011; Carranza 2015: 27). Ésto permitirá evaluar el potencial de desplazamiento y observar si el mismo reviste características particulares como la reorientación de las piezas, (cambios en la posición) y vincularlo con el volumen de las mismas (Carranza et al. 2014).

Del mismo modo servirá para estimar la probabilidad de enterramiento a lo largo del esquema diacrónico planteado y establecer si efectivamente existen puntos de acumulación de materiales con la consiguiente formación de palimpsestos. Como se mencionó anteriormente el área fue ocupada desde el 3000 A.P. en adelante, pero la reciente recuperación en superficie y estratigrafía de tiestos cerámicos en la costa continental (inmediatamente próxima al Islote) señala que la circulación humana pudo producirse en diferentes momentos. En tal sentido es posible esperar que la acción de los pingüinos en el Islote esté incrementando la mezcla de materiales líticos diacrónicos.

Si bien ya se había señalado para otros sectores litorales de Patagonia la perturbación de los depósitos arqueológicos (concheros) por la formación de áreas de nidificación de pingüinos de Magallanes -como por ejemplo en Punta Medanosas, Monte León y Cabo Vírgenes (Caracotche et al. 2005; Cruz 1999; 2004; 2008), los estudios tafonómicos se centraron en generar criterios útiles para evaluar la conformación de los conjuntos óseos que incluyeran este taxón en cada caso (Cruz 2007). Creemos que este caso, muestra el potencial de una perspectiva tafonómica lítica, útil para evaluar los procesos de formación en depósitos arqueológicos costeros afectados por la acción tafonómica de las aves marinas en áreas de nidificaciones actuales. Estos estudios permiten explorar la dinámica y continua transformación, permitiendo plantear nuevas preguntas sobre los contextos de deposición y la conformación de los materiales líticos en superficie, que es necesario establecer previo a entregar interpretaciones sobre el registro arqueológico y a la forma en que los grupos humanos del pasado utilizaron el espacio litoral.

Por último, las observaciones tafonómicas realizadas, así como la distribución del registro lítico en el espacio estudiado, sugieren esta vía de análisis como un aporte para evaluar la acción tafonómica de los pingüinos en conjuntos de superficie. 


\section{Agradecimientos}

Los trabajos de investigación en la costa de Río Negro son financiados por el CONICET (PIP No 112-201101-00589) y Agencia FONCyT (PICT No 1128) Argentina. A la Agencia Cultura de Río Negro y Áreas Naturales Protegidas, que otorgaron los permisos correspondientes para poder realizar los trabajas en este sector de la costa Patagónica. Agradecemos especialmente a Jimena Alberti por su ayuda con el resumen en inglés. A Nelson Iribarren y familia por su apoyo durante los trabajos de campo en la zona.

\section{Referencias}

Alberti, J. 2015. Análisis de la disponibilidad y explotación de fuentes de materias primas líticas durante el Holoceno medio y tardío en la costa del golfo San Matías, provincia de Río Negro, Argentina. Un enfoque regional. Tesis Doctoral inédita. Facultad de Filosofía y Letras. Universidad de Buenos Aires, Buenos Aires, 324 p. (in Spanish) ("Availability and use of sources of lithic raw materials during the middle and late Holocene in the San Matías Gulf Coast, Río Negro, Argentina: A regional approach”)

Aschero, C. 1975. Ensayo para una clasificación morfológica de artefactos líticos aplicada a estudios tipológicos comparativos. Informe presentado al CONICET. MS., Buenos Aires, 113 p. (in Spanish) ("Reviewing typological issues regarding bifacial artifacts classification”)

Aschero, C. 1983. Ensayo para una clasificación morfológica de artefactos líticos. Apéndice A y B. Cátedra de Ergología y Tecnología. Facultad de Filosofía y Letras, Universidad de Buenos Aires, Buenos Aires, 180 p. (in Spanish) ("Reviewing typological issues regarding bifacial artifacts classification”)

Bertellotti, M. 2013. Pingüino de Magallanes. Embajador de Patagonia. Vázquez Mazzini Editores, Buenos Aires, 59 p. (in Spanish) ("Magallanes Penguin, Ambassador of Patagonia”)

Borella, F., Scartascini, F, \& Marani, H. 2011. Explorando la subsistencia humana a partir de la evidencia faunística en la costa rionegrina. En: Arqueología de pescadores y marisqueadores en Nordpatagonia. Descifrando un registro de más de 6000 años, (Borella, F. \& Cardillo, M., Eds.), Editorial Dunken, Buenos Aires: p. 87-110. (in Spanish) ("Exploring human subsistence from faunal evidence in the rio negro coast")

Borella, F. \& Borrero L. A. 2010. Observaciones tafonómicas acerca de la desarticulación de carcasas de pinnípedos en ambientes litorales, el caso de Islote Lobos (Golfo San Matías, Río Negro). En: Zooarqueología a principios del siglo XXI: aportes teóricos, metodológicos y casos de estudio. (Gutiérrez, M. A., De Nigris, M., Fernández, P. M., Giardina, M., Gil, A. F., Izeta, A., Neme, G. \& Yacobaccio, H. D., Eds.), Libros del Espinillo, Buenos Aires: p. 371-379. (in Spanish) ("Taphonomic observations concerning dismantling of seal carcasses in coastal environments, Islote Lobos (San Matías Gulf, Río Negro)”)

Borrazzo, K. 2006. Tafonomía lítica en dunas: una propuesta para el análisis de los artefactos líticos. Intersecciones en Antropología, 7: 247-261. (in Spanish) ("Lithic taphonomy in dunes: a proposal for the lithic artifacts analysis”)

URL: http://www.scielo.org.ar/scielo.php?script=sci_abstract\&pid=S1850373X2006000100018 
Borrazzo, K. 2011. Tafonomía lítica en la estepa patagónica: experimentación y registro arqueológico de superficie. En: Bosques, montañas y cazadores: investigaciones arqueológicas en Patagonia Meridional, (Borrero, L. \& Borrazzo, K. Eds.) CONICETIMHICIHU, Buenos Aires: p. 127-153. (in Spanish) ("Lithic taphonomy in the steppe: experimentation and the surface archaeological record")

Borrero, L. 2004. Tafonomía regional: el caso de los pinnípedos. Contra viento y marea. Arqueología de la Patagonia, (Civalero. M.T., Fernández, P.M. \& Guraieb A.G., Eds.) Instituto Nacional de Antropología y Pensamiento Latinoamericano y Sociedad Argentina de Antropología, Buenos Aires: p. 445-454. (in Spanish) ("Regional taphonomy: the pinnipeds case”)

Brown, D. \& Rothery, P. 1993. Models in biology: mathematics, statistics and computing. John Wiley \& Sons Ltd., Chichester, 688 p. (en inglés; in English) ("Modelos en biología: Matemáticas, estadística y computación”)

Burroni, D., Donahue, R., Pollard, M. \& Mussi, M. 2002. The Surface Alteration Features of Flint Artefacts as a Record of Environmental Processes. Journal of Archaeological Science, 29: 1277-1287. (en inglés; in English) ("Las características de alteración superficial de artefactos de sílex como un registro de los procesos ambientales”) doi:10.1006/jasc.2001.0771

Butler, D. R. 1995. Zoogeomorphology. Animals as geomorphic agents. Cambridge University Press, Cambridge, 231 p. (en inglés; in English) (“Zoogeomorfología. Los animales como agentes geomorfológicos”)

Camuffo, D. 1995. Physical Weathering of Stones. The Science of the Total Environment, 167: 4-14. (en inglés; in English) (“La degradación física de las rocas”) doi:10.1016/0048-9697(95)04565-I

Caracotche, M.S., Cruz, I., Espinoza, S., Carballo Marina, F. \& Belardi, J.B. 2005. Rescate arqueológico en el Parque Nacional Monte León (Santa Cruz, Argentina). Magallania, 33(2): 143-163. (in Spanish) (“Archaeological rescue in Monte Leon (Santa Cruz, Argentina) National Park”). doi:10.4067/S0718-22442005000200010

Carranza, E. 2015. Análisis de conjuntos líticos de superficie desde una perspectiva tafonómica. Estudios en la costa norte del golfo San Matías, Río Negro, Argentina. Tesis de Licenciatura inédita. Facultad de Filosofía y Letras, Universidad de Buenos Aires, Buenos Aires, 114 p. (in Spanish) (“Analysis of surface lithic assemblages from a taphonomic perspective. Studies from San Matías gulf, Río Negro, Argentina”)

Carranza, E., Cardillo, M., Alberti, J. \& Favier Dubois, C. (2014), Taphonomic studies of surface lithic scatters from the coastal area of San Matías Gulf, Río Negro province, Argentina. Poster at the 4th Southern Deserts Conference. Laboratorio de PaleoEcología Humana, Facultad de Ciencias Exactas y Naturales, Universidad Nacional de Cuyo, Mendoza. (en inglés; in English) ("Los estudios tafonómicos de las dispersiones líticas de superficie en la zona costera del Golfo San Matías, provincia de Río Negro, Argentina”) 
Cardillo, M. \& Favier Dubois, C. 2011. Una aproximación al uso del espacio en la Costa Norte del Golfo San Matías (Río Negro, Argentina): Relaciones entre la evidencia artefactual e isotópica. En: Movilidad y Migraciones. III Jornadas Interdisciplinarias de Historia y Ciencias Humanas, (Guiance, A., Ed.), CONICET - IMHICIHU, Buenos Aires: p. 241-252. (in Spanish) ("An approach to the use of space in the northern coast of San Matías Gulf ( Río Negro, Argentina): relations between artifactual and isotopic evidence”)

Cardillo, M. \& Scartascini. F. 2007. Tendencias observadas en las estrategias de explotación de recursos líticos en el Golfo de San Matías, provincia de Río Negro, Argentina. En: Arqueología de Fuego-Patagonia. Levantando piedras, desenterrando huesos... y develando arcanos (Morello, F., Martinic, M., Prieto, A. \& Bahamonde, G., Eds.), CEQUA, Punta Arenas: 117-127. (in Spanish) ("Trends in the exploitation strategies of lithic resources in San Matias gulf, Río Negro Province, Argentina”)

Cruz, I. 1999. Pingüinos de cabo Vírgenes (Santa Cruz). Aspectos tafonómicos e implicaciones arqueológicas. Actas del XIII Congreso Nacional de Arqueología Argentina (Gomez Otero, J., Ed.), Universidad Nacional de Córdoba, Tomo 4, Cordoba: p. 95-108. (in Spanish) ("Penguins in Vírgenes cape (Santa Cruz). taphonomic aspects and archaeological implications”)

Cruz, I. 2004. Tafonomía de huesos de aves en Punta Medanosa (Depto. Puerto Deseado, Santa Cruz, Argentina). En: Contra viento y marea. Arqueología de la Patagonia, (Civalero. M.T., Fernández, P.M. \& Guraieb A.G., Eds.) Instituto Nacional de Antropología y Pensamiento Latinoamericano y Sociedad Argentina de Antropología, Buenos Aires: p. 455-468. (in Spanish) ("Taphonomy of bird bones in Punta Medanosa (Depto. Puerto Deseado, Santa Cruz, Argentina”)

Cruz, I. 2007. Avian Taphonomy: Observations at two Magellanic penguin (Spheniscus magellanicus) breeding colonies and their implications for the fossil record. Journal of Archaeological Science, 34: 1252-1261. (en inglés; in English) ("La tafonomía de aves: Las observaciones en dos colonias reproductivas de Pingüinos magallánicos (Spheniscus magellanicus) y sus implicaciones para el registro fósil”) doi:10.1016/j.jas.2006.10.016

Cruz, I. 2008. Avian and mammalian bone taphonomy in southern continental Patagonia: A comparative approach. Quaternary International, 180: 20-27. (en inglés; in English)

("Tafonomía ósea de huesos de mamíferos y aves en el sur de la Patagonia continental: Un enfoque comparativo”) doi:10.1016/j.quaint.2007.08.008

Dorn, R. 2009. Desert Rock Coatings. En: Geomorphology of Desert Environments, (Parsons, A. \& Abrahams, A., Eds.) Springer, New York: p. 153-186. (en inglés; in English) (“Geomorfología de ambientes desérticos”) doi:10.1007/978-1-4020-5719-9

Favier Dubois, C. 2013. Hacia una cronología del uso del espacio en la costa norte del golfo San Matías (Río Negro, Argentina): Sesgos geológicos e indicadores temporales. En: Tendencias teórico-metodológicas y casos de estudio en la arqueología de la Patagonia, (Zangrando, A., Barberena, R., Gil, A., Neme, G., Giardina, M., Luna, L., Otaola, C., Paulides, S., Salgán, L. \& Tívoli, A. Eds.) Museo de Historia Natural de San Rafael-INAPL, Buenos Aires: p. 87-96 (in Spanish) (“Towards a chronology of space use in the coast of the San Matías gulf (Río Negro, Argentina): geological biases and temporal indicators") 
Favier Dubois, C. \& Borella, F. 2011. Contrastes en la costa del golfo: una aproximación al estudio del uso humano del litoral rionegrino en el pasado. En: Arqueología de pescadores y marisqueadores en Nordpatagonia. Descifrando un registro de más de 6000 años, (Borella, F. \& Cardillo, M. Eds.), Editorial Dunken, Buenos Aires: p. 13-42. (in Spanish) ("Differences in the coast of the Gulf: an approach to the study of the human use of the Río Negro province coast")

Favier Dubois, C., Borella, F. \& Tykot. R. 2009. Explorando tendencias en el uso humano del espacio y los recursos en el litoral rionegriono (Argentina) durante el Holoceno medio y tardío. En: Arqueología de la Patagonia - Una mirada desde el último confín (Salemme, M., Santiago, F., Álvarez, M., Piana, E. Vázquez, M. \& Mansur, E. Eds.) Utopías, Ushuia: p. 985-997. (in Spanish) ("Exploring trends in the human use of space and resources in the coast of Río Negro province (Argentina) during Middle and Late Holocene”)

Favier Dubois, C. \& Scartascini, F. 2012. Intensive fishery scenarios on the North Patagonian coast (Río Negro, Argentina) during the Mid-Holocene. Quaternary International, 256: 62-70. (en inglés; in English) ("Escenarios de la pesca intensiva en la costa norte de la Patagonia (Río Negro, Argentina) durante el Holoceno Medio”) doi:10.1016/j.quaint.2011.07.041

González, P., Bertellotti, M., Giaccardi, M., Lini, R., Lizurume, M.E. \& Yorio, P. 1998. Distribución reproductiva y abundancia de las aves marinas de Río Negro. En: Atlas de la distribución reproductiva de Aves Marinas en el Litoral Patagónico Argentino (Yorio, P., Frere, E., Gandini, P. \& Harris, G., Eds.) Fundación Patagonia Natural, Buenos Aires: p. 29-37. (in Spanish) ("Reproductive distribution and abundance of marine birds of Río Negro coast ”)

Hammer, Ø., Harper, D. \& Ryan, R. 2001. PAST - Palaeontological Statistics software package for education and data analysis. Palaeontologica Electronica, 4(1): 1-9. (en inglés; in English) ("PAST - Software de estadística paleontológica para educacion y análisis de datos”) URL: http://palaeo-electronica.org/2001_1/past/past.pdf

Manzi, L.M., Borella, F. \& Cardillo, M. 2011 Distribuciones artefactuales: una aproximación a la estructura espacial del registro arqueológico del litoral atlántico rionegrino. En: Arqueología de pescadores y marisqueadores en Nordpatagonia. Descifrando un registro de más de 6000 años (Borella, F. \& Cardillo, M., Eds.), Editorial Dunken, Buenos Aires: p. 43-66. (in Spanish) (“Artifactual distributions: an aproximation to spatial estructure of the archaeological record of Rio Negro Atlantic coast”)

Sánchez, L. 1973, Geología litoral. In: Relevamiento Ecológico y tipificación de las comunidades del Litoral Marítimo de la Provincia de Río Negro, con especial referencia al establecimiento de áreas de cultivo para especies de interés comercial, Instituto de Biología Marina, Rio Negro: p. 112-141. (in Spanish) ("Ecological survey and classification of the Maritime Coastal communities of the Río Negro province, with particular reference to the establishment of cultivation areas for commercial species")

Schiavini, A., Yorio, P., Gandini, P. A., Raya Rey, A., \& Boersma, P.D. 2005. Los pingüinos de las costas argentinas: estado poblacional y conservación. Hornero, 20(1): 5-23. (in Spanish) ("Penguins of Argentine coast: population status and conservation"). URL: http://www.scielo.org.ar/scielo.php?script=sci_abstract\&pid=S007334072005000100002 
Stokes, D.L. \& Boersma, P.D. 1991. Effects of substrate on the distribution of Magellanic penguin (Spheniscus magellanicus) burrows. The Auk, 108: 923-33. (en inglés; in English) ("Efectos del sustrato sobre la distribución de las madrigueras de pingüinos Magallánicos (Spheniscus magellanicus)”) Stable

URL: http://www.jstor.org/stable/4088322

Schiffer, M. 1983. Toward the Identification of Formation Processes. American Antiquity, 48(4): 675-706. (en inglés; in English) ("Hacia una identificación de los procesos de formación”) doi:10.2307/279771

Svendsen, G., Romero, M., Borella, F. \& González, R. 2009. Informe del relevamiento de los apostaderos de lobos marinos de un pelo, Otaria flavescens, de la provincia de Río Negro durante enero de 2009. Instituto de Biología Marina y Pesquera "Almirante Storni”, San Antonio Oeste, Río Negro, 130 p. (in Spanish) ("Survey report of the one hair sea lions Otaria flavescens, in the Rio Negro Province in January 2009”) 


\title{
Taphonomy and lithic technology in a highly dynamic environment: The case of the penguin colony of La Pastosa Islet (Río Negro Province), Patagonia, Argentina
}

\author{
Marcelo Cardillo $^{1}$, Eugenia Carranza ${ }^{2}$, Florencia Borella ${ }^{3}$ \\ 1. IMICHIHU Consejo Nacional de Investigaciones Científicas y Técnicas. Saavedra 15. 5to piso. Facultad de \\ Filosofía y Letras. Universidad de Buenos Aires (UBA). Buenos Aires, Argentina. \\ Email: marcelo.cardillo@gmail.com \\ 2. Facultad de Filosofía y Letras. Universidad de Buenos Aires (UBA), Puán 480, Buenos Aires, Argentina. \\ Email: carranza.e89@gmail.com \\ 3. INCUAPA-CONICET. Facultad de Ciencias Sociales, Universidad Nacional de Centro de la Provincia de \\ Buenos Aires (UNICEN), Argentina. Avenida Del Valle 5737 (7400), Olavarría, Buenos Aires, Argentina. \\ Email: fborella@soc.unicen.edu.ar
}

\begin{abstract}
:
This paper presents the first results of taphonomic and technological analyses of the lithic assemblage recovered on La Pastosa Islet, on the coast of Río Negro province, Patagonia, Argentina. This archaeological record is particular because it has been under a high formational dynamic and is located in an area recently occupied by a colony of Magellan penguins (Spheniscus magellanicus). Two samplings of lithic material were made, for which technological, metric and taphonomic variables were registered.

La Pastosa is an 8 hectare islet, which is located $768 \mathrm{~m}$. from the continent, and is only attached to it at low tide by a narrow land strip that runs through a marsh. While the absolute age of this island is unknown, we know that from the late Holocene, around $3000 \mathrm{BP}$, sea level reached current levels (Favier Dubois 2013), and since that time the islet was visited by hunter-gatherers, who buried their dead in the highest part of the island (Favier Dubois et al. 2007, Favier Dubois et al. 2009). Today, this area has a great biodiversity, such as a reproductive colony of sea lions (Otaria flavescens) which has recently formed (Svendsen et al. 2009: 8) as well as a variety of seabirds.

Artifact sampling was focused on the area of greatest density of lithic artifacts, currently close to the center of the islet (Figure 1). The context of recovery of these materials can be divided into three categories: A) common transit areas of penguins that give shape to paths free of vegetation; B) burrow entrances C) marginal spaces that correspond to sectors of debris accumulation (twigs and dry grass, feathers, Penguin carcasses) on the margins of the transit area (Figure 2).

During sampling, the original position of the artifacts, or face of recovery, and context of discovery were recorded. At the lab, general typological (tool, core, or debitage), raw materials, metrics (volume in $\mathrm{mm}^{3}$ ), and taphonomic variables were registered. The latter in particular were used to characterize and compare both assemblages.

The recording and analysis of artifact modification due to taphonomic processes is a line of research to approach the intensity of the physical transformations in the archaeological context, and to indicate their degree of integrity (Schiffer 1983; Burroni et al. 2002). The variables recorded were as follows, the level of weathering in each tool face (W: W0, W1, W2, W3); the presence of carbonate concretion; and the integrity of the piece (whole or broken).

The results show that intensive penguin traffic and burrow activity exposed buried materials in certain sectors (Figure 5.1), which are pushed through paths with no vegetation (Figure 5.2) and redeposited in "fall off" areas with little or no circulation (Figure 5.3). These are mostly small patches of vegetation and organic waste, which have high relative stability. For this reason we believe that in
\end{abstract}


these patches, materials are more likely to accumulate and perhaps re-enter into the substrate. Preliminary evidence obtained for this study area suggests that the lithic record is in continuous formation, in this case because of the action of penguins. Since the establishment of this colony is recent, its impact on the archaeological record is significant.

Keywords: North Patagonian coast; lithic taphonomy; penguin; natural formation processes; La Pastosa Islet 